\title{
THE BAIRE CATEGORY AND FORCING LARGE LINDELÖF SPACES WITH POINTS $G_{\delta}$
}

\author{
ISAAC GORELIC
}

(Communicated by Andreas R. Blass)

\begin{abstract}
For $\kappa$ as large an aleph as we want, we construct by forcing a model in which $\mathrm{CH}$ holds and there is a Lindelöf zero-dimensional space of size $\kappa$ with points $G_{\delta}$.
\end{abstract}

In 1922 (published 1929) Alexandroff and Urysohn proved, for Hausdorff Lindelöf spaces $X$, that if closed sets are $G_{\delta}$ then $|X| \leq \mathfrak{c}$. They asked whether it is sufficient to assume only that $X$ has countable character [1].

In 1969 Arhangel'skii showed that this is indeed the case [2]. And he, in turn, asked whether countable pseudo-character (points are $G_{\delta}$ sets) is enough.

In 1978 Shelah gave a consistent answer $N O$ to the question of Arhangel'skii by constructing a model of $\mathrm{CH}$ and in it a Lindelöf space with points $G_{\delta}$ of size $\mathrm{c}^{+}=\aleph_{2}[3,4]$. This was the best result up to this point. The question of the true limit of the size of Lindelöf spaces with points $G_{\delta}$ was still unanswered. In particular, it was an open problem to find, consistent with $\mathrm{CH}$, such a space of size $\aleph_{3}[4]$.

In this paper we build for any cardinal $\lambda$ a model in which $2^{\aleph_{0}}=\aleph_{1}, 2^{\aleph_{1}}>\lambda$ and there is a Lindelöf, 0-dimensional Hausdorff space, with points $G_{\delta}$ and cardinality $2^{\aleph_{1}}$. The problem of whether consistently there can be such spaces of size larger than $2^{\aleph_{1}}$ remains open.

Assume, without loss of generality, that $V \models \mathrm{CH} \& \kappa^{\aleph_{1}}=\kappa$. Fix a disjoint partition of $\kappa$ into countable infinite sets and its enumeration $\left\{A_{\alpha}: \alpha<\kappa\right\}$, and, for each $\alpha$ in $\kappa$, a countable set $H_{\alpha}$ dense in $2^{A_{\alpha}}, H_{\alpha}=\left\{h_{\xi}: \xi \in A_{\alpha}\right\} \subset$ $2^{A_{\alpha}}$.

Notation. For $F \subset 2^{\kappa}$ and $K \subset \kappa$, let $F \uparrow K:=\{f \uparrow K: f \in F\}$. For $s \in$ $F n\left(\kappa, 2, \kappa^{+}\right)$, let $U_{s}:=[s]:=\left\{f \in 2^{\kappa}: s \subset f\right\}$. Hence, for example, $U_{s} \uparrow K=$ $\left\{f\{K: s \subset f\} \subset 2^{K}\right.$. If $B \subset F n(A, 2)$, let $U_{B}:=\bigcup\left\{U_{s}: s \in B\right\}$.

Let $p \in \mathbb{P}$ iff $p=\langle F, T>$ and there are (necessarily unique) $I, A, G$ satisfying the following conditions (and then we also write $\langle I, A, G, F, T\rangle=$ $\left.\left\langle I^{p}, A^{p}, G^{p}, F^{p}, T^{p}\right\rangle\right)$ :

(1) $I \in[\kappa] \leq \omega$;

(2) $A=\bigcup\left\{A_{\alpha}: \alpha \in I\right\}$;

(3) $G=\left\{g_{\alpha}: \alpha \in I\right\}$ and $(\forall \alpha \in I) g_{\alpha}: A \backslash A_{\alpha} \rightarrow 2$;

Received by the editors May 20, 1991 and, in revised form, October 23, 1991.

1991 Mathematics Subject Classification. Primary 03E35, 54D20, 54B10. 
(4) $F=\left\{f_{\xi}: \xi \in A\right\} \subset 2^{A}$ and $(\forall \alpha \in I)\left(\forall \xi \in A_{\alpha}\right)\left(f_{\xi}\left\lceil\left(A \backslash A_{\alpha}\right)=g_{\alpha}\right.\right.$ and $\left.f_{\xi} \mid A_{\alpha}=h_{\xi}\right)$.

Remark. So $f_{\xi}=g_{\alpha} \cup h_{\xi}$ and $(\forall \alpha \in I)\left\{f_{\xi}: \xi \in A_{\alpha}\right\}$ is a dense subset of $U_{g_{\alpha}} \mid A$. The set $U_{g_{\alpha}} \mid A$ is a closed $G_{\delta}$ (hence nowhere dense, if $|I|>1$ ) in $2^{A}$, but of second category in itself, because it is isomorphic to the Cantor set $2^{A_{\alpha}}$

(5) $(\forall \alpha \in I)(\forall \beta \in I \backslash\{\alpha\})\left(\forall \xi \in A_{\alpha}\right) g_{\beta} \mid A_{\alpha} \neq h_{\xi}$.

Remark. Therefore $(\forall \alpha \in I)\left(\forall \xi \in A_{\alpha}\right)(\forall \eta \in A \backslash\{\xi\}) f_{\xi}\left|A_{\alpha} \neq f_{\eta}\right| A_{\alpha}$, and so $A_{\alpha}$ witnesses that $\left\{f_{\xi}\right\}$ will be a $G_{\delta}$, for every $\xi$ in $A_{\alpha}$.

(6) $|T| \leq \omega$ and $(\forall B \in T) B \subset F n(A, 2)$ with $F \subset U_{B} \mid A:=\bigcup\left\{U_{s} \mid A\right.$ : $s \in B\}$, i.e., $(\forall \xi \in A)(\exists s \in B)$ with $s \subset f_{\xi}$.

Remark. In other words, $B$ codes an open cover $U_{B} \mid A$ of $F \subset 2^{A}$. Hence, by (4), $(\forall B \in T)(\forall \alpha \in I) U_{B} \mid A$ is dense open in $U_{g_{\alpha}} \mid A$. We say $q \leq p$ in $\mathbb{P}$ iff

(a) $I^{q} \supset I^{p}$,

(b) $\left(\forall \xi \in A^{p}\right) f_{\xi}^{q}\left\lceil A^{p}=f_{\xi}^{p}\right.$, and

(c) $T^{q} \supset T^{p}$.

Note. (b) is equivalent to $\left(\forall \alpha \in I^{p}\right) g_{\alpha}^{q}\left\lceil A^{p}=g_{\alpha}^{p}\right.$.

Fact 1. $(\forall p \in \mathbb{P})(\forall \alpha \in \kappa)(\exists q \leq p)$ with $\alpha \in I^{q}$. Hence $(\forall \xi \in \kappa) \quad D_{\xi}:=\{q \in$ $\left.\mathbb{P}: \xi \in A^{q}\right\}$ is dense in $\mathbb{P}$.

Fact 2. $\mathbb{P}$ is countably closed, moreover, $\omega_{1}$-complete.

Fact 3. $\mathbb{P}$ has the $\omega_{2}$-chain condition.

Definition. Let $G$ be a $\mathbb{P}$-generic filter over $V$. For every $\xi \in \kappa$, let $f_{\xi}:=$ $\bigcup\left\{f_{\xi}^{p}: p \in G \& \xi \in A^{p}\right\}$. Let $F:=\left\{f_{\xi}: \xi \in \kappa\right\}$

Fact 4. In $V[G], 2^{\omega}=\omega_{1}, 2^{\omega_{1}}=\kappa$, all cardinals are preserved. Also $(\forall \xi \in \kappa)$ $f_{\xi}: \kappa \rightarrow 2$ is a total function and $F$ is a points $G_{\delta}$ subspace of $2^{\kappa}$, in particular, $|F|=\kappa$.

Fact 5. In $V[G], F$ is Lindelöf.

The proof of the facts will involve no combinatorics.

Fact 1 is true by the Baire Category Theorem. Without loss of generality, $\alpha \notin I^{p}$.

If $I^{p}=\varnothing$, let $q:=\left\langle H_{\alpha}, \varnothing\right\rangle$. Otherwise, pick $\beta \in I^{p}$ and find, by the Baire Category Theorem,

$$
g_{\alpha} \in\left(U_{g_{\beta}} \mid A^{p}\right) \cap \bigcap\left\{U_{B} \mid A^{p}: B \in T^{p}\right\} \backslash\left\{f_{\xi}: \xi \in A_{\beta}\right\} \neq \varnothing .
$$

Also pick $h_{*} \in 2^{A_{\alpha}} \backslash H_{\alpha}$. Now let $I^{q}:=I^{p} \cup\{\alpha\}$ and, for every $\gamma \in I^{p}$, set $g_{\gamma}^{q}:=g_{\gamma}^{p} \cup h_{*}$, and let $G^{q}:=\left\{g_{\gamma}^{q}: \gamma \in I^{p}\right\} \cup\left\{g_{\alpha}\right\}$ and $T^{q}:=T^{p}$.

Fact 2 is obvious.

Fact 3 is true by the Baire Category Theorem: Suppose $\mathbb{Q} \subset \mathbb{P}$ and $|\mathbb{Q}|=\omega_{2}$. We will show that we can find two conditions, $p$ and $q$, in $\mathbb{Q}$ that can be extended to some $r \leq p, q$ in $\mathbb{P}$.

By $\mathrm{CH}$, the $\Delta$-system lemma and simple counting we may assume that there are $p \neq q$ in $\mathbb{Q}$ that are isomorphic structures, i.e., that $\operatorname{tp} I^{p}=\operatorname{tp} I^{q}, \operatorname{tp} A^{p}=$ 
tp $A^{q}$, and denoting by $\xi^{\prime}$ the unique ordinal in $A^{q}$ corresponding to a $\xi \in A^{p}$ such that $\operatorname{tp}\left(A^{p} \cap \xi\right)=\operatorname{tp}\left(A^{q} \cap \xi^{\prime}\right)$, we have $\left(\forall \xi \in A^{p}\right)\left(\forall \eta \in A^{p}\right) f_{\xi}^{p}(\eta)=f_{\xi^{\prime}}^{q}\left(\eta^{\prime}\right)$, and, moreover, $\forall x \in \Delta:=A^{p} \cap A^{q}\left(=\bigcup\left\{A_{\alpha}: \alpha \in I^{p} \cap I^{q}\right\}\right) f_{\xi}^{p}(x)=f_{\xi^{\prime}}^{q}(x)$.

We shall construct an $r \in \mathbb{P}$ with $r \leq p, q$ of the form $r=\left\langle I^{p} \cup I^{r}, A^{p} \cup\right.$ $\left.A^{q}, F^{r}, G^{r}, T^{p} \cup T^{q}\right\rangle$. For every $\xi \in A^{p}, f_{\xi}^{r}$ will be an extension of $f_{\xi}^{p}$ on $A^{q} \backslash \Delta$, and for every $\xi^{\prime} \in A^{q}, f_{\xi^{\prime}}^{r}$ will extend $f_{\xi^{\prime}}^{q}$ on $A^{p} \backslash \Delta$. Of course, we need to extend, appropriately, only $g_{\alpha}$ 's.

There are three cases.

Case 1. $\alpha \in I^{p} \cup I^{q}$. Set $g_{\alpha}^{r}:=g_{\alpha}^{p} \cup g_{\alpha}^{q}$.

Case 2. $\alpha \in I^{p} \backslash I^{q}$. By the Baire Category Theorem, there is an $h: A^{q} \rightarrow 2$ with

$$
h \in\left(U_{g_{\alpha^{\prime}}}\left\lceil A^{q}\right) \cap \bigcap\left\{U_{B} \mid A^{q}: B \in T^{q}\right\} \backslash\left\{f_{\eta}^{q}: \eta \in A_{\alpha^{\prime}}\right\} \neq \varnothing .\right.
$$

Then let $g_{\alpha}^{r}:=g_{\alpha}^{p} \cup h$.

Case 3. $\alpha^{\prime} \in I^{q} \backslash I^{p}$. This is the same as Case 2. There is an $h: A^{p} \rightarrow 2$ with

$$
h \in\left(U_{g_{\alpha}} \mid A^{p}\right) \cap \bigcap\left\{U_{B} \mid A^{p}: B \in T^{p}\right\} \backslash\left\{f_{\eta}^{p}: \eta \in A_{\alpha}\right\} .
$$

Set $g_{\alpha \prime}^{r}:=g_{\alpha \prime}^{q} \cup h$. That $r$ thus defined belongs to $\mathbb{P}$ (and, hence, extends both, $p$ and $q$ ) is immediate.

All of Fact 4, except $\kappa \leq 2^{\omega_{1}}$, follows from Facts 1, 2, and 3; and $\kappa \leq 2^{\omega_{1}}$ follows, for example, from the observation that $\left\{g_{\alpha} \uparrow \omega_{1}: A_{\alpha} \cap \omega_{1}=\varnothing\right\}$ is an enumeration of distinct functions on $\omega_{1}$.

Proof of Fact 5. First observe that if $p \in \mathbb{P}$ and $B \in T^{p}$, then $p \| \vdash \check{U}_{B}$ covers $\dot{F}$ ". Indeed, for every $\xi \in \kappa, D(B, \xi):=\left\{q \in \mathbb{P}: q \| \dot{F}(\xi) \in \check{U}_{B}\right\}$ is dense below $p$ by Fact 1 (and because $\left\{q \in \mathbb{P}: q \leq p \& \xi \in A^{q}\right\} \subset D(B, \xi)$ ).

Let $\sigma$ be a $\mathbb{P}$-name for a basic open cover (in $2^{\kappa}$ ) of $\dot{F}$, and let some $p$ force this, i.e., $p \Vdash$ “ $\sigma: \check{\kappa} \longrightarrow \check{F} n(\kappa, 2) \&((\forall \xi \in \kappa) \sigma(\xi) \subset \dot{F}(\xi)) . "$

It is enough to show that there is an extension $q \leq p$ that decides the values of $\sigma(\xi)$ for all $\xi \in A^{q}$ and such that the domains of these decided functions $\sigma(\xi)$ all lie inside $A^{q}$.

That is, we want $q \leq p$ and $B=\left\{s_{\xi}: \xi \in A^{q}\right\} \subset F n\left(A^{q}, 2\right)$ with $q \mid \vdash$ " $\left(\forall \xi \in \check{A^{q}}\right) \sigma(\xi)=\check{s}_{\xi} "$. Then we may let $q^{*}:=\left\langle F^{q}, T^{q} \cup\{B\}\right\rangle$ with $q \geq q^{*} \in \mathbb{P}$. And then, by our observation above, $q^{*} \mid \vdash$ " $\sigma \mid \check{A}^{q^{*}}=\check{B}$ and covers $\dot{F}$."

Thus we would establish the density of such conditions below a member of $G$, hence the existence of such $q^{*}$ in $G$, hence our Fact. But such $q \leq p$ exists because $\mathbb{P}$ is $\omega_{1}$-complete.

To wit, $\forall \bar{p} \leq p$ and $\forall \xi \in A^{\bar{p}} \exists r \leq \bar{p}$ that decides $\sigma(\xi)$ (since $\sigma$ is mapping into $V$ ), so $\exists s_{\xi} \in F n(\kappa, 2)$ with $r \Vdash \sigma(\check{\xi})=\check{s}_{\xi}$; and, by Fact 1 , we may assume that $s_{\xi} \subset A^{r}$. Then, "closing up" and using Fact 2 , we may see that there is a $q \leq p$ we want.

Remarks. 1. As $\mathbb{P}$ stands, there are $p \in \mathbb{P}$ which force $\dot{F}$ not to be dense in $2^{\kappa}$. For example, a $p \in \mathbb{P}$ with $I^{p}=\{0,1\}$ and

$$
T^{p}=\left\{\left\{\left\{\left\langle\xi_{0}, g_{1}\left(\xi_{0}\right)\right\rangle\right\},\left\{\left\langle\xi_{1}, g_{0}\left(\xi_{1}\right)\right\rangle\right\}\right\}\right\},
$$

where $\xi_{0}=$ an ordinal in $A_{0}$ and $\xi_{1}=$ some ordinal in $A_{1}$. Then $p$ “ $\dot{U}_{\left\{\left\langle\xi_{0}, 1-g_{1}\left(\xi_{0}\right)\right\rangle,\left\langle\xi_{1}, 1-g_{0}\left(\xi_{1}\right)\right\rangle\right\}} \cap \dot{F}=\varnothing$." 
2. But with a slightly more work, we can make $\dot{F}$ dense in $2^{\kappa}$, and hence, e.g., c.c.c. For example, we could additionally require that, for $p \in \mathbb{P}, F^{p}$ be dense in $2^{A^{p}}$; or, alternatively, that for every $B$ in $T^{p}, U_{B} \uparrow A^{p}$ be dense in $2^{A^{p}}$ (and then the density of $\dot{F}$ would follow by genericity).

3. An unessential modification of $\mathbb{P}$ will yield in the extension $V\left[G^{\prime}\right]$ two Lindelöf spaces (with points $G_{\delta}$ ) $F$ and $F^{\prime}$ such that their product $F \times F^{\prime}$ has a closed discrete subspace of size $\kappa=2^{\aleph_{1}}$. As a consequence, in $V\left[G^{\prime}\right]$, $2^{\aleph_{1}}$ is "subbase Lindelöf." Indeed, let ${H^{\prime}}_{\alpha}:=\left\{h_{\xi}^{\prime}: \xi \in A_{\alpha}\right\}$, where $h_{\xi}^{\prime}$ । $\left(A_{\alpha} \backslash\{\xi\}\right):=h_{\xi} \uparrow\left(A_{\alpha} \backslash\{\xi\}\right)$ and $h_{\xi}^{\prime}(\xi):=1-h_{\xi}(\xi)$. Define, for $\xi \in A_{\alpha}$, $f_{\xi}^{\prime}:=g_{\alpha} \cup h_{\alpha}^{\prime}, F^{\prime}:=\left\{f_{\xi}^{\prime}: \xi \in A\right\}$ and force with (the conditions containing) two sets of covers, $T$ of $F$ and $T^{\prime}$ of $F^{\prime}$, simultaneously. This works, because $H_{\alpha}^{\prime}$ again is dense in $2^{A_{\alpha}}$. Then, in the generic extension, $\left\{\left\langle f_{\xi}, f_{\xi}^{\prime}\right\rangle: \xi<\kappa\right\}$ is a closed discrete subspace of $F \times F^{\prime}$. And since its two projections are Lindelöf, it is "subbase Lindelöf."

4. If $\aleph_{1}<\lambda<\kappa$ with $\operatorname{cf}(\lambda)>\omega$, then adding $\lambda$ Cohen reals to $V[G]$ (i.e., forcing from $V[G]$ with $F n(\lambda, 2))$ we are getting a model in which $2^{\aleph_{0}}=\lambda>$ $\aleph_{1}$ and our space $F$ is still Lindelöf (see, e.g., [5, Lemma 3.3]). This answers another consistency question.

5. The Lindelöf property of $F$ can be destroyed by a simple c.c.c. forcing. Indeed, take any $\alpha \in \kappa$ such that $A_{\alpha} \cap \omega_{1}=\varnothing$. Then $\forall \gamma<\omega_{1}$

$$
D_{\gamma}:=\left\{p \in \mathbb{P}:\left(\exists \xi \in \kappa \backslash A_{\alpha}\right) p \| \dot{f}_{\xi}\left\lceil\gamma=\dot{g}_{\alpha}\lceil\gamma\}\right.\right.
$$

is dense in $\mathbb{P}$ (use the proof of the Fact 1 ). Hence in $V[G]$ there is a set of size $\omega_{1}$

$$
S=\left\{f_{\xi_{\gamma}}: \gamma<\omega_{1}\right\} \text { s.t. } \forall \gamma<\omega_{1} f_{\xi_{\gamma}} \mid \gamma=g_{\alpha}\lceil\gamma \text {. }
$$

If $f \in F$ is a complete accumulation point (CAP) of $S$, then $f\left\lceil\omega_{1}=g_{\alpha} \uparrow\right.$ $\omega_{1}$. Therefore, $f \in\left\{f_{\xi}: \xi \in A_{\alpha}\right\}$. Let $C=$ the set of all CAPs of $S$, then $\bar{C}=C \subset\left\{f_{\xi}: \xi \in A_{\alpha}\right\}$. Therefore $|C| \leq \aleph_{0}$ and $\Psi(C, F)=\aleph_{1}$.

Now force the Martin's Axiom $M A_{\omega_{1}}$ from our $V[G]$ as a ground model. Extending an argument in [6], we have $M A_{\omega_{1}} \Rightarrow \mathfrak{b}>\aleph_{1} \Rightarrow$ "Countable closed subsets in a Lindelöf space with points $G_{\delta}$ cannot have pseudocharacter exactly $\aleph_{1} . "$ (In general, $\Psi(C, F) \notin\left[\aleph_{1}, \mathfrak{b}\right)$.) Therefore, in the resulting model of $M A_{\omega_{1}}, S$ has a subset of size $\aleph_{1}$ without a CAP in $F$, and so $F$ is not Lindelöf (it has an open cover of size $\aleph_{1}$ without a subcover of a smaller size).

\section{ACKNOWLEDGMENT}

The author is very grateful to Professor Franklin Tall for posing this question to him in 1988, to Professor William Weiss for helping to write this paper, and to Miss Soňa Kunová for both helping and hindering.

\section{REFERENCES}

1. P. S. Alexandroff and P. S. Urysohn, Memoire sur les espaces topologiques compacts, Nederl. Akad. Wetensch. Proc. Ser. A 14 (1929), 1-96.

2. A. V. Arhangel'skii, On the cardinality of bicompacta satisfying the first axiom of countability, Soviet Math. Dokl. 10 (1969), 951-955.

3. S. Shelah, On some problems in general topology, preprint, 1978. 
4. I. Juhasz, Cardinal functions. II, Handbook of Set-Theoretic Topology (K. Kunen and J. E. Vaughan, eds.), North-Holland, Amsterdam, 1984.

5. A. Dow, Two applications of reflection and forcing to topology, Proc. Sixth Prague Topological Symposium, Helderman Verlag, Berlin, 1986.

6. M. Bell and J. Ginsburg, First countable Lindelöf extensions of uncountable discrete space, Canad. Math. Bull. 23 (1980).

Department of Mathematics, University of Toronto, Toronto, Ontario, Canada M5S $1 \mathrm{Al}$

Current address: Institute of Mathematics and Computer Science, The Hebrew University of Jerusalem, Givat Ram, 91904 Jerusalem, Israel 